\title{
Optimization for Time-driven Link Sleeping Reconfigurations in ISP Backbone Networks
}

\author{
Frederic Francois, Ning Wang, Klaus Moessner and Stylianos Georgoulas \\ Centre for Communication Systems Research \\ University of Surrey \\ Guildford GU2 7XH, UK \\ \{f.francois, n.wang, k.moessner, s.georgoulas\}@surrey.ac.uk
}

\begin{abstract}
Backbone network energy efficiency has recently become a primary concern for Internet Service Providers and regulators. The common solutions for energy conservation in such an environment include sleep mode reconfigurations and rate adaptation at network devices when the traffic volume is low. It has been observed that many ISP networks exhibit regular traffic dynamicity patterns which can be exploited for practical time-driven link sleeping configurations. In this work, we propose a joint optimization algorithm to compute the reduced network topology and its actual configuration duration during daily operations. The main idea is first to intelligently remove network links using a greedy heuristic, without causing network congestion during off-peak time. Following that, a robust algorithm is applied to determine the window size of the configuration duration of the reduced topology, making sure that a unified configuration with optimized energy efficiency performance can be enforced exactly at the same time period on a daily basis. Our algorithm was evaluated using on a Point-ofPresence representation of the GÉANT network and its real traffic matrices. According to our simulation results, the reduced network topology obtained is able to achieve $18.6 \%$ energy reduction during that period without causing significant network performance deterioration. The contribution from this work is a practical but efficient approach for energy savings in ISP networks, which can be directly deployed on legacy routing platforms without requiring any protocol extension.
\end{abstract}

Keywords-green network, energy efficiency, traffic engineering, link sleeping, network reconfiguration

\section{INTRODUCTION}

The energy consumption of modern computer networks has become an issue of concern for both Internet Service Providers (ISPs) and regulators. European Telecoms currently consume 21.4TWh per year and this is expected to increase to $35.8 \mathrm{TWh}$ by 2020 if no green networking technologies are implemented [1]. Backbone ISP networks currently consume around $10 \%$ of the total network power requirements but this figure will jump to $40 \%$ by 2017 [2] if no green actions are taken. This is due to the increasing use of bandwidth-hungry applications such as Video-on-Demand (VoD) and cloud computing over the Internet.

In recent years, various green techniques have been proposed in the research community towards future energyaware ISP network platforms. Existing energy-efficiency paradigms can be classified into both "disruptive" and evolutionary solutions. On one hand, design of green networking architectures, mechanisms and protocols has been advocated, which aims to realize intrinsic support of future energy-aware ISP networks ([3][4][5][6]). On the other hand, green network management paradigms can be also applied on top of legacy systems even without any fundamental modification of the underlying network platforms ([7][8]), with a typical example being energy-aware traffic engineering (ETE) techniques. While disruptive approaches aim at longterm solutions for greening the future Internet, short or midterm evolutionary schemes are of equal significance, as the environmental issue and the scarcity of energy resources are already regarded as imminent threats.

In this work, our proposed approach falls into the ETE paradigm which encompasses all techniques that are used to optimize traffic flows with the ultimate objective of reducing the network power consumption, but without causing traffic congestion due to the reduced network capacity. ETE is a relatively new topic of research which was first introduced in [9], and comprehensive introduction and analysis of ETE paradigms can be found in [10] and [11]. Effectively, ETE paradigms can be further classified into offline and online techniques.

Most research works in literature have followed the offline optimization strategy $([12][13][14][15])$ because of its simplicity compared to online energy saving methods ([9][16][17]). Global optimization methods used in the case of offline TE are more predictable in terms of network behavior than the local optimization methods used for online intradomain TE [18]. Offline ETE approaches ([12][19]) aim to compute a reduced network topology which is optimized for a static traffic matrix (TM) only. Despite their simplicity, offline approaches are not able to adaptively cope with traffic dynamics, which is something common in operational ISP networks. To circumvent this problem, complimentary online ETE solutions have also been proposed which aim to enable adaptive traffic control against changing traffic conditions ([9][16][17]). However, online paradigms need much more complicated monitoring and control intelligence, and hence existing approaches are mainly confined to a theoretical study level.

To overcome the aforementioned difficulties, we propose a practical and efficient approach with time-driven network topology reconfigurations. The rationale behind this is that many ISP networks have regular/predictive traffic behavior patterns ([20][21][22]) which offers the opportunity for simple 
network topology control rather than relying on complicated online ETE paradigms. Our proposed algorithm aims to compute a reduced network topology which will give the optimized combination of number of sleeping links and the duration of the sleeping period. This energy-saving approach can be used because networks are currently dimensioned and operated by taking into consideration traffic demands during peak hours and are further overprovisioned to account for unexpected events. Therefore, there is room for reducing the network topology during the off-peak time and save energy while still maintaining the same degree of over-provisioning that occurs during peak time network operation. The reduced topology can be directly enforced based on existing multitopology routing protocols such as MT-OSPF [23] and MTISIS [24]. In order to obtain a robust off-peak network reconfiguration, the calculation of the off-peak topology and its duration is based on multiple sampled traffic matrices as input, and the detailed description of the algorithm will be given in sections III and IV.

As far as simulation-based experiments are concerned, we use the GÉANT network topology - which consists of 23 nodes and 74 links - together with its historical traffic matrices over five days for performance evaluation. The results show that our proposed algorithm is able to achieve significant energy savings for a variety of constraint policies on the maximum allowable link utilization and traffic matrix scaling factor. For instance, $18.6 \%$ energy savings over 5 days can be achieved when the allowable Maximum Link Utilization (MLU) constraint in the off-peak window is set to $80 \%$. In this case, 33 links out of the total 74 links can be reconfigured to sleep mode, and the daily off-peak time duration using the reduced network topology configuration is 10 hours. Such energy savings are achieved through a simple time-driven network reconfiguration operation, but without imposing the need for any additional intelligence or complexity to the network itself.

\section{RELATED WORK}

Reduction of power consumption in computer networks can be achieved through several strategies. The first strategy is the use of green hardware for networking devices; at the physical level more energy-efficient circuit design can be used ([25][26][27]), and at the functional level dynamic frequency and voltage scaling can be employed ([28][29][19]). Secondly, it is also possible to make use of green networking protocols. Green protocols can allow, for example, proxies to take over the functionality of the network devices that will be put into sleep mode ([12][30][22]). Such an approach has been illustrated in [22] where a router can transparently transfer all the virtual links from one line card to another so that the former can be put to sleep. Another example is to modify the OSPF protocol by making a subset of routers use the routing table of other routers rather than calculating their own, so that fewer active links are used [12]. The third strategy is the use of green network management paradigms, such as ETE, where traffic demands are routed appropriately in order to promote either link rate adaptation $([16][17][31])$ and/or link sleeping
([13][12][14]) by taking into consideration the energy consumption characteristics of links and line cards. Future energy savings due to the exploitation of different possible energy consumption profiles of links have also been investigated in [19] and [32].

\section{SCHEME OVERVIEW}

According to the proposed scheme, two distinct network topologies are used in daily operation. During the normal operation hours, the original full topology is applied for handling customer traffic, as it happens in common practice. During off-peak time, the reduced network topology that excludes scheduled sleeping links is used for energy saving purposes. In order to seamlessly perform topology switching without incurring routing disruption, we propose to use multitopology routing protocols ([24][23]) as the underlying routing platform. Specifically, two routing topologies are configured, one with the full physical topology and the other excluding the links scheduled to sleep. Once the operation has entered the scheduled off-peak time, individual routers simultaneously activate the reduced routing topology by remarking the multitopology identifier (MT-ID) of packets from the default full topology MT-ID to the reduced topology MT-ID. Similarly, when the scheduled off-peak time expires, all routers remark customer packets back to the full topology MT-ID. Such operation avoids the rigid routing re-convergence procedure based on one single topology, which is generally considered to be harmful. From an optimality point of view, a technical challenge of having these two network topologies is that the reduced network topology should not be exclusively optimized with respect to the number of sleeping links, but it should also support as long operational periods as possible with this reduced network capacity, but without causing network congestion incurred due to customer traffic dynamics. It is not difficult to infer that there is a tradeoff which needs to be optimized, between the number of sleeping links in the reduced topology and the duration of the off-peak time when this topology is actually applied. This is because, if an excessive number of links is excluded from the network based on a purely greedy approach, then the resulting reduced topology is not able to handle even minor traffic increases, and in this case the full topology has to be restored, which leads to very short duration for the use of the reduced topology.

In order to determine the optimized combination of the reduced topology and its applied operation duration, it is important to consider how traffic behavior patterns can play a role. As such, network monitoring is periodically performed which produces distinct traffic matrices at regular time intervals (e.g. every tens of minutes), as is the case in operational networks (GÉANT [33] and Abilene networks [34]). It should be noted that network configurations based on one single traffic matrix are not sufficiently robust in dealing with traffic dynamics, as this has been indicated in conventional traffic engineering paradigms [10]. Therefore, we consider multiple traffic matrices as input in order to produce the optimized combination of the reduced topology and its off-peak operation duration. To ease the time-driven 
reconfiguration operations, it is also desired that the off-peak configuration starts at exactly the same time and has the same duration on a daily basis (e.g. 7:00PM-7:00AM). According to our proposed solution, a single synthetic traffic matrix is computed based on multiple sampled traffic matrices across a given period (e.g. weekly). These selected traffic matrices represent the traffic pattern during the daily off-peak period. Thereafter, an oblivious reduced network topology with the excluded sleeping links is computed based on the single synthetic traffic matrix. Similarly, determining the off-peak duration on a daily basis also takes into account traffic matrix patterns during each day. It is worth mentioning that, although traffic dynamics behaviors exhibit some similar patterns on a daily basis, it is also important to make sure the same off-peak topology can be applied in a unified time-driven manner even though there can be traffic pattern variations at the same (offpeak) time in each day. Input traffic matrices to our algorithm can also be scaled up so as to enhance the robustness of the calculated reduced network topology to potential changes in the traffic patterns. In the next section, we will first formulate the overall optimization problem, followed by joint schemes of computing the reduced topology and determining its duration. The outcome is an optimized network topology coupled with a unified time window for its configuration on a daily basis.

\section{PROBLEM FoRMULATION AND PROPOSED ALGORITHM}

\section{A. Problem Formulation}

TABLE I. DEFINITION OF SYMBOLS

\begin{tabular}{|l|l|}
\hline Variable & Description \\
\hline$A_{z}$ & $\begin{array}{l}\text { Set of sleeping links in the reduced topology during } \\
\text { off-peak time }\end{array}$ \\
\hline$I_{z}$ & $\begin{array}{l}\text { Set of consecutive traffic matrices supported with } \\
A_{z}\end{array}$ \\
\hline$G(V, E)$ & $\begin{array}{l}\text { Directed graph with } V \text { being set of nodes and } E \\
\text { being set of links }\end{array}$ \\
\hline$c_{i j}$ & Bandwidth capacity of link from node $i$ to $j$ \\
\hline$t^{s d}$ & Traffic demand from node $s$ to $d$ \\
\hline$f_{i j}^{s d}$ & $\begin{array}{l}\text { Traffic demand from } s \text { to } d \text { that traverses link from } \\
i \text { to } j\end{array}$ \\
\hline$f_{i j}$ & Total traffic demand on link from $i$ to $j$ \\
\hline$\alpha$ & $\begin{array}{l}\text { Maximum allowable fractional utilisation of link } \\
\text { capacity }\end{array}$ \\
\hline$P\left(t^{s d}\right)$ & Set of links taken by $t^{s d}$ from node $s$ to $d$ \\
\hline
\end{tabular}

The overall problem formulation on optimizing the reduced topology and its duration can be expressed as:

$$
\operatorname{maximize} \max \left\{\left|I_{z}\right| \times\left|A_{z}\right|\right\}
$$

subject to:

$$
\sum_{j=1}^{|V|} f_{i j}^{s d}-\sum_{j=1}^{|V|} f_{j i}^{s d}=\left\{\begin{array}{cl}
t^{s d} & \forall s, d, i=s \\
-t^{s d} & \forall s, d, i=d \\
0 & \forall s, d, i \neq s, d
\end{array}\right.
$$

$$
\begin{gathered}
f_{i j}<\alpha c_{i j} \quad \forall i, j \\
P\left(t^{s d}\right) \cap I_{z}=\emptyset \quad \forall s, d, z
\end{gathered}
$$

Equation (1) is the objective function which jointly optimizes the reduced topology (in terms of the number of sleeping links) and the duration of its configuration (in terms of the number of consecutive traffic matrices which can be supported by that topology). The overall off-peak time duration can be calculated from the number of traffic matrices, which are captured at regular intervals, covered by the period. Constraint (2) is the standard flow conservation constraint. Constraint (3) ensures that all links have utilization below a given threshold determined by the ISP. That is, with the reduced topology, the maximum link load should not exceed the threshold $\alpha$ (in terms of the fraction of the link capacity) at any time during the off-peak time. Constraint (4) makes sure that only the active links can carry the traffic during the offpeak time period. In addition, it is also required that the reduced network topology should not be broken due to the link removals, and also on a daily basis the starting/ending time for the off-peak topology configuration should be unified.

\section{B. Proposed Heuristic Algorithm}

Fig. 1 illustrates the overall approach. The curve in the figure indicates the actual maximum link utilization (MLU) dynamics pattern across a given period $\left(5\right.$ days $\left.^{1}\right)$. Such an MLU curve is effectively plotted according to the monitored traffic matrices at a certain time interval. In this example, we illustrate that with the TM measurements taking place every 2 hours, 12 TMs are produced within each 24 hour period. In Fig. $1, M_{w, n}$ indicates the $n^{\text {th }}$ TM in day $w$. Such historical TM information is used for computing future off-peak network configurations and this is analogous to the forecasted traffic matrix used in general traffic engineering. First of all, multiple "sampled" TMs from different days in the considered period are used for computing a synthetic off-peak TM, based on which sleeping links are initially identified in the reduced network topology. The purpose of using multiple TMs instead of a single one is for robustness reasons, as traffic conditions even at the same time point in different days may vary. In addition, in order to compute the actual off-peak configuration period (time window) on a daily basis, a unified starting point for computation on each day is be identified, i.e. this point is located at the same $n$ for every day $w$. Starting from this point, an algorithm is designed for expanding (in both directions) the off-peak configuration window size incrementally in order to maximize the overall energy savings based on the reduced offpeak topology. As the final result, the reduced topology can be configured in a unified way on a daily basis, for instance

${ }^{1}$ To better illustrate the daily off-peak configuration windows, we purposely start from midday on the first day with peak traffic volume in the network. Also it is not necessary for the peak/valley MLU to occur exactly at the same time point on each day. This figure is only for illustration purposes. 
between traffic matrix $M_{w, n 1}$ and $M_{w, n 2}$ within each $w^{\text {th }}$ day. For instance, in Fig. 1 the values of $\mathrm{n} 1$ and $\mathrm{n} 2$ for identifying the unified daily off-peak time are 4 and 7 respectively.

The overall heuristic-based approach consists of the following three sequential stages: (1) computing the synthetic traffic matrix and the starting point for off-peak window size expansion, (2) greedy link removal for the provisionally reduced topology, and (3) the joint expansion of the off-peak window size and the finalization of the reduced topology to be applied during the off-peak period.

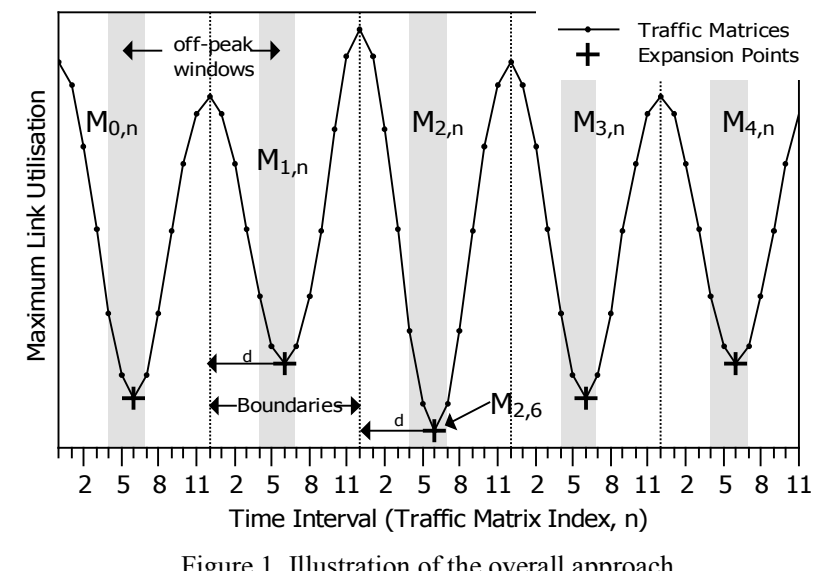

1) Stage 1: Computing the synthetic TM and the starting point for off-peak window size expansion

As previously indicated, the off-peak operation window size can be represented with a sequence of TMs at regular intervals, for instance every 2 hours as shown in Fig. 1. The MLU of each traffic matrix is calculated to identify the traffic matrix with the lowest MLU across the whole period (denoted as TM*) together with its "location" in the sequence of the TMs (e.g. TM $M_{2,6}$ in Fig. 1). The $2^{\text {nd }}$ index of this traffic matrix with the lowest MLU (i.e. 6 in this specific example) is set to be the expansion point of the daily off-peak window. To enable a unified daily off-peak window, the location of the expansion point in every other day is at the same position as $\mathrm{TM}^{*}$. This is represented in Fig. 1 through the calculation of distance $d$ from the beginning of each day. Put in other words, since $\mathrm{TM}^{*}$ in the figure is $M_{2,6}$, then the expansion point in other days should be $M_{w, 6}$ (i.e. $d=6$ ), where $w$ represents the index of the days under consideration. It is worth mentioning that we do not assume the actual lowest MLU always takes place exactly at the expansion point in the other days. But this is not exhibited in Fig. 1 simply for simplicity reasons. These expansions points are the starting points that will be used in the $3^{\text {rd }}$ stage to determine the actual location and the size of the off-peak windows.

As for the traffic matrix information to be used for computing the reduced topology with link removal, it is not efficient if only one single sampled TM is used as input, say $\mathrm{TM}^{*}$ with the lowest MLU across the whole period. A more robust strategy is to take into account multiple TMs in order to obtain an optimized network configuration across all scenarios during the period. As such, we first compute a synthetic traffic matrix based on individual TMs located on the daily expansion points. In this example, the overall synthetic TM is computed according to the 5 TMs shown in Fig 1. For each traffic volume entry in the synthetic TM, the value is effectively the average of the corresponding entries from the 5 input TMs at the daily expansion points.

\section{2) Stage 2: Greedy link removal}

In this stage, a reduced network topology is provisionally computed based on the synthetic TM obtained from the previous stage. Further fine-tuning of this topology will be performed jointly with the determination of the actual off-peak operational window size in the next stage. The computation of this provisional reduced topology is achieved by the iterative greedy removal of the links from the full network topology. The detailed operation of the link removal in this stage is shown in Fig. 2.

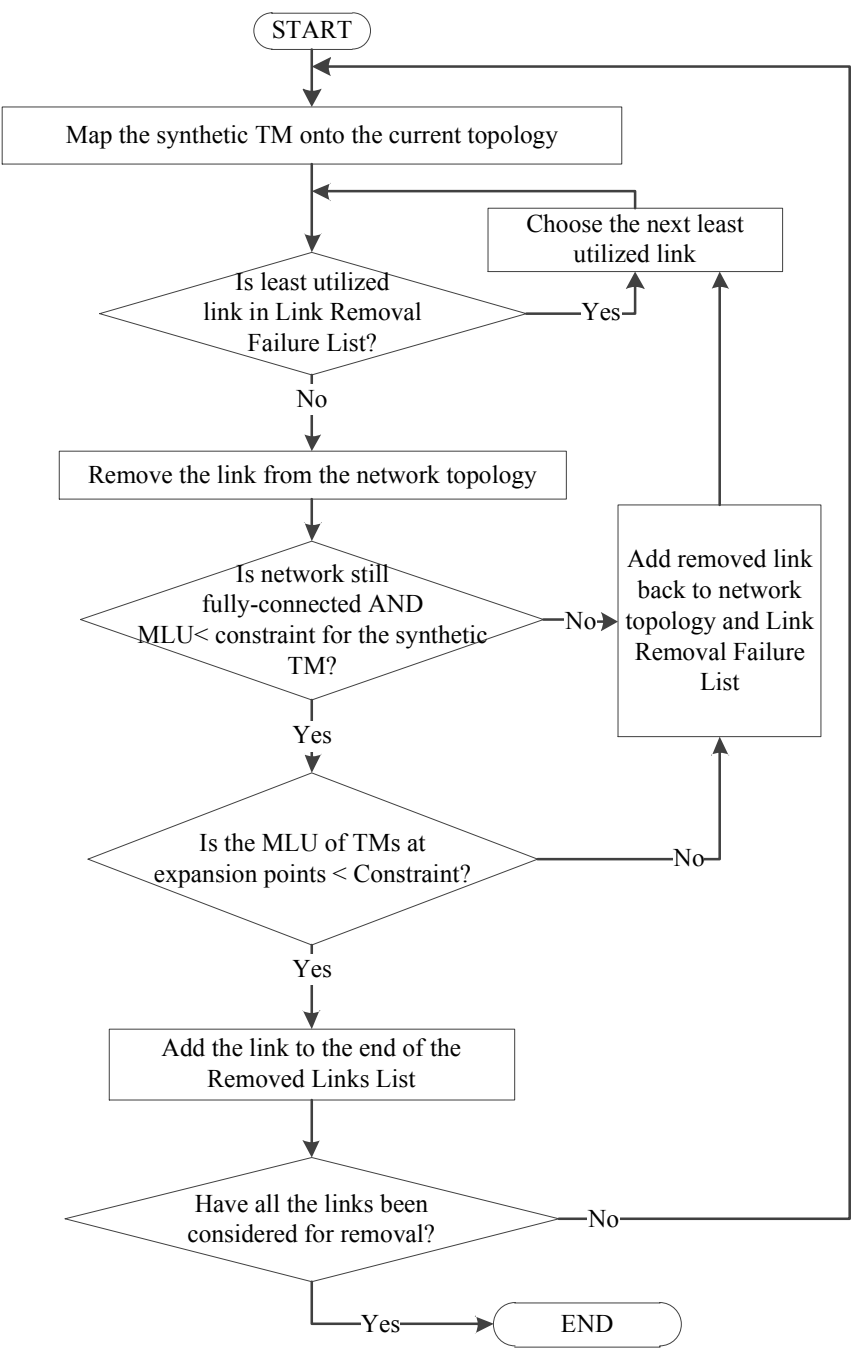

Figure 2. Flow chart for greedy link removal.

Specifically, upon the actual removal of any link, the corresponding utilization of all the links is updated in the whole residual topology. If the link under consideration cannot be removed, it is put into the Link Removal Failure List so that 
it will not be further considered. The condition for a successful link removal is that the residual network topology still remains connected and also the resulting MLU does not exceed the predefined constraint $\alpha$ based on the input synthetic TM. In addition, considering the requirement that the daily off-peak windows need to have unified starting time and duration, for robustness concerns the traffic matrices at all expansion points are also tested for compliance with the constraints (3) and (4) (shown in section IV. A) each time one link is considered for removal from the network topology. At the end of this operation, the network topology with least remaining active links, which still satisfies (3) and (4), is determined as the reduced topology. This topology will be further refined jointly with the optimization of the off-peak time window size in the next stage.

3) Stage 3: Determination of off-peak time window size and the final reduced topology

The final and main stage of the algorithm is to compute the actual off-peak window size jointly with the fine-tuning of the number of sleeping links. The flow chart in Fig. 3 shows the detailed operation in this stage. To start, the maximum number of consecutive TMs - which can be supported by the reduced network topology computed in the previous stage-is identified. The expansion of the daily off-peak windows starts from the expansion points obtained in Stage 1, which is identified by $M_{w, b}$. From this point the expansion is performed in both directions sequentially and independently. This is because the traffic behavior pattern is generally not "symmetric" in the two directions from the expansion points. In each direction, if the projected MLU of the next TM does not exceed the predefined constraint for all considered days, then the time duration between that next TM and the current one can be included in the off-peak time window.

After the maximum number of consecutive TMs is obtained, the algorithm reconsiders the possibility of restoring some sleeping links from the reduced topology computed in Stage 2 and checks if this leads to an improved value of the overall objective function. The consideration of adding sleeping links is in the reverse order by which the links were removed from the network topology in the previous stage. This step is necessary because it is possible to have a sufficient increase in the number of consecutive TMs supported (i.e. enlarged off-peak time window size) to compensate for a reduced number of sleeping links and therefore, obtain better performance according to the objective function.

The termination condition for the iterative consideration of restoring sleeping links depends on the best value of the objective function so far. The algorithm stops if it reaches a point where the number of sleeping links is not sufficient to improve the best value of the objective cost function even if the entire time period is considered off-peak.

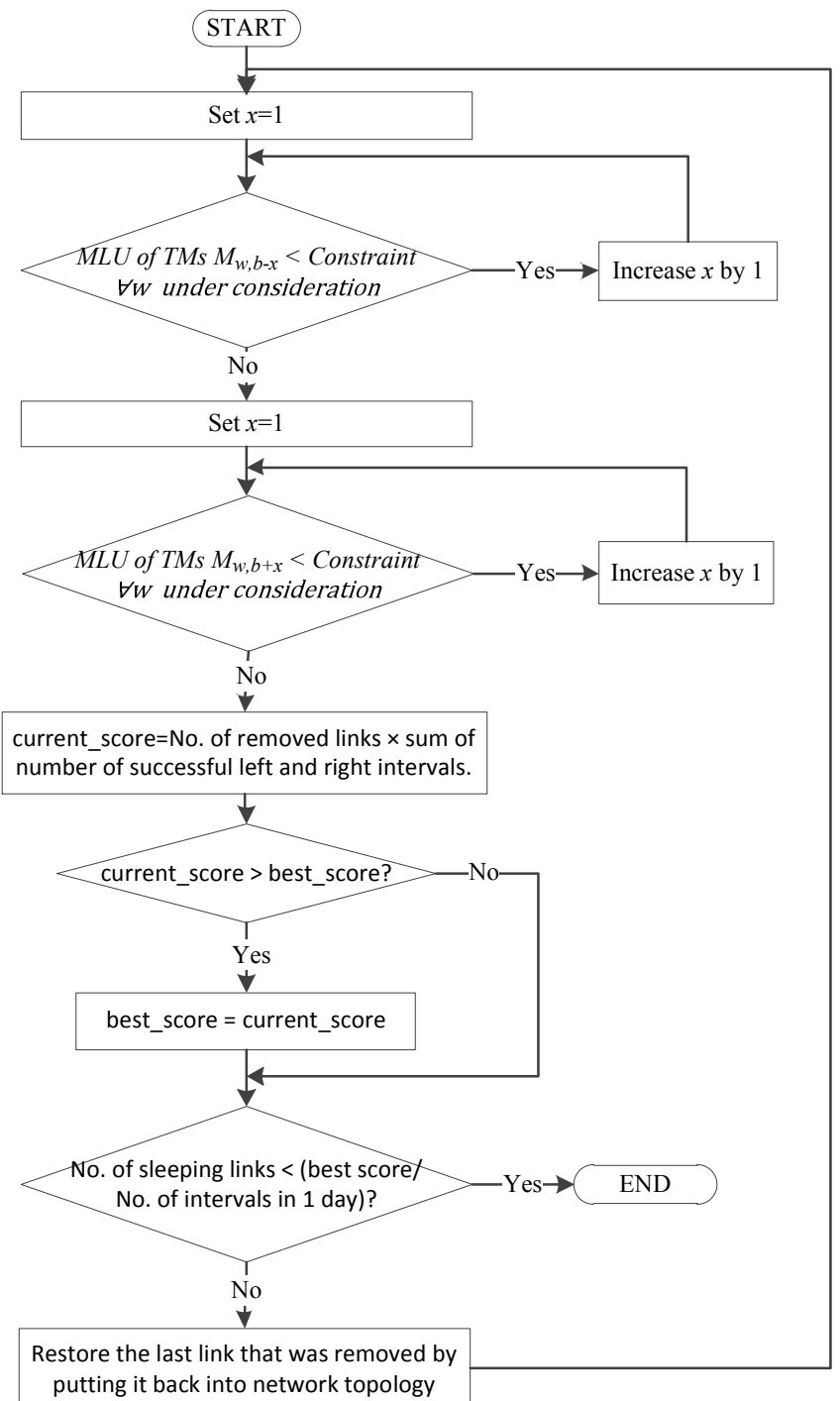

Figure 3. "Off-peak window determination" stage flowchart.

\section{PERFormace EVAluation}

\section{A. Experiment Setup}

We evaluate the proposed scheme based on the operational GÉANT network topology and its published traffic traces. The topology consists of 23 Points-of-Presence (PoPs) and 74 unidirectional links of varying bandwidth capacity.

As far as the operational duration is concerned, we consider 480 consecutive traffic matrices at 15-minute intervals from Monday midday to Saturday midday according to the historical GÉANT traffic matrix data set. The statistical characteristics of the traffic matrices during this period are given in Table II.

As previously mentioned, the network operator may have different policies in setting the constraint threshold for the maximum allowable link load based on the reduced topology during the off-peak time. This is effectively reflected by the value of $\alpha$ in Inequality (3) in the problem formulation. In our simulation experiment, we consider different values for $\alpha$ which represent the conservativeness in the link removal operation. A higher value of $\alpha$ indicates that higher MLU is 
tolerable and in this case, more energy can be conserved due to either increased number of links to be removed and/or expanded off-peak time duration allowed. In our experiment, we consider the following 3 values for $\alpha$ : $0.6,0.7$ and 0.8 , meaning that the maximum allowable MLU is $60 \%, 70 \%$ and $80 \%$ respectively. Certainly the constraint can be further relaxed (i.e. to further increase the value of $\alpha$ ), however this may lead to increased vulnerability of the reduced network topology, especially due to unexpected traffic upsurge during the off-peak operational period. In addition, we also scale up the actual traffic demand in the TMs by $10 \%$ to $30 \%$ in order to evaluate the energy efficiency with higher traffic volume scenarios. The aim is to make sure that the reduced network topology can also tolerate some variation in MLU values because of traffic uncertainly. The scaling factor is represented by $X$.

TABLE II. CHARACTERISTICS OF SET OF TRAFFIC MATRICES

\begin{tabular}{|l|l|}
\hline MLU & Value (\%) \\
\hline Max. & 90.9 \\
\hline Min. & 30.9 \\
\hline Mean & 58.6 \\
\hline $1^{\text {st }}$ Quartile & 44.5 \\
\hline $2^{\text {nd }}$ Quartile & 55.9 \\
\hline $3^{\text {rd }}$ Quartile & 74.2 \\
\hline
\end{tabular}

\section{B. Simulation Results}

The proposed scheme was evaluated with different constraint thresholds $(\alpha)$ and traffic volume scaling factors $(X)$ as shown in Table III. In the table, $O$ is the total off-peak time duration (in terms of minutes) per 24-hour operation and $Y$ represents the energy savings (in terms of percentage) over the whole operation period under consideration.

It can be observed from Table III that, as the MLU constraint becomes more stringent, the off-peak window size and number of sleeping links decrease. The window size decreases more significantly compared to the decrease in the number of sleeping links with this specific traffic pattern. This phenomenon is more noticeable when the traffic matrices are scaled up and the MLU constraint is kept constant. The most important result from Table III is that the proposed algorithm is able to achieve $18.6 \%$ energy savings over 5 consecutive working days while having a maximum MLU constraint of $80 \%$. With more conservative threshold settings, smaller energy savings can be achieved due to the shrunk off-peak window size and/or reduced number of sleeping links.

It is also interesting to investigate the change of end-to-end delay based on the reduced topology. Table IV shows the average increase in delay in the off-peak window where the reduced network topology is applied. The calculation of delay in the GÉANT network is based on its IGP link weight settings, as the configuration of IGP weights is according to the link delay [21]. According to our results, the average delay experiences only a $3.2 \%$ increase when the MLU constraint is set at $80 \%$ (without traffic volume scaling). The increase in delay under the other MLU constraints is very close to the $80 \%$ scenario because the number of sleeping links is the same (the delay values are the same in Table IV due to reduction of accuracy when rounding off). The only change is the duration of the off-peak windows which does not significantly affect the delay performance in this scenario.

TABLE III. ENERGY SAVINGS OF PROPOSED ALGORITHM

\begin{tabular}{|l|l|l|l|l|}
\hline $\boldsymbol{\alpha}$ & $\boldsymbol{X}$ & $\left|\boldsymbol{A}_{\mathbf{Z}}\right|$ & $\boldsymbol{O}$ & $\boldsymbol{Y}(\boldsymbol{\%})$ \\
\hline 0.8 & 1 & 33 & 600 & 18.6 \\
\cline { 2 - 5 } & 1.1 & 33 & 450 & 13.9 \\
\cline { 2 - 5 } & 1.2 & 33 & 375 & 11.6 \\
\cline { 2 - 5 } & 1.3 & 33 & 360 & 11.1 \\
\hline 0.7 & 1 & 33 & 435 & 13.5 \\
\cline { 2 - 5 } & 1.1 & 33 & 360 & 11.1 \\
\cline { 2 - 5 } & 1.2 & 33 & 315 & 9.76 \\
\cline { 2 - 5 } & 1.3 & 32 & 405 & 12.2 \\
\hline 0.6 & 1 & 33 & 330 & 10.2 \\
\cline { 2 - 5 } & 1.1 & 32 & 420 & 12.6 \\
\cline { 2 - 5 } & 1.2 & 32 & 405 & 12.2 \\
\cline { 2 - 5 } & 1.3 & 32 & 375 & 11.3 \\
\hline
\end{tabular}

TABLE IV. DELAY INCREASE DUE TO REDUCED TOPOLOGY

\begin{tabular}{|l|l|}
\hline $\boldsymbol{\alpha}$ & \% increase in delay metric in window \\
\hline 0.8 & 3.20 \\
\hline 0.7 & 3.20 \\
\hline 0.6 & 3.20 \\
\hline
\end{tabular}

Figs. 4, 5 and 7 show the actual MLU performance with time across the 5 considered days for different $\alpha$ constraint values. During the peak-time operation period (outside the dark area), the MLU performance is based on the full network topology. There are two MLU curve segments in the off-peak duration: the solid curves represent the MLU performance based on the actual reduced topology with the exclusion of sleeping links, while the dashed curves indicate the expected MLU performance based on the original full topology. Our observation on the MLU comparison is as follows: at first thought, the MLUs in the off-peak windows are expected to be higher when the reduced network topology is used rather than the full network topology. This is because the number of links available to carry the traffic demands is lower in the former scenario which uses the reduced topology. An interesting observation, however, is that there are also cases where the full network topology gives a higher MLU compared to the reduced network topology. This is because the greedy link removal operation of our proposed algorithm may remove a link which originally injects traffic into the most loaded link of the network based on the full topology. The removal of such a link diverts some customer traffic away from that most loaded link to other alternate paths.

This scenario is illustrated with the example in Fig. 6 and Table V. The figure shows a simple topology in which the links have different capacities, and the IGP link weights are in inverse proportion to the link capacities. Table $\mathrm{V}$ shows the traffic matrix information to be mapped onto the topology. Initially, there is no link removal and the traffic demand from node $A$ to $C$ is routed through node $B$ and it consumes 10 units of bandwidth on the link B-C, which is the most loaded link in 
the network. When link A-B is removed from the topology, then the traffic demand from $A$ to $C$ will be diverted on to the path A-D-C. The link utilization increase on links A-D and D$\mathrm{C}$ due to the diverted traffic is not enough to make one of them the most loaded link in the network. Although link B-C remains the most loaded link, its load is reduced and hence the maximum MLU in the network is reduced.

It should be noted that the original IGP link weight settings in the GÉANT network are not optimized for traffic engineering [21], and this explains why our proposed algorithm is able to further reduce the MLUs in the off-peak windows even with a reduced topology. Overall, the proposed greedy link removal algorithm is very efficient, as it is able to reconfigure 33 out of 74 links to sleep mode during the offpeak time in most experiments shown in Table III, which represents more than $40 \%$ of the links.

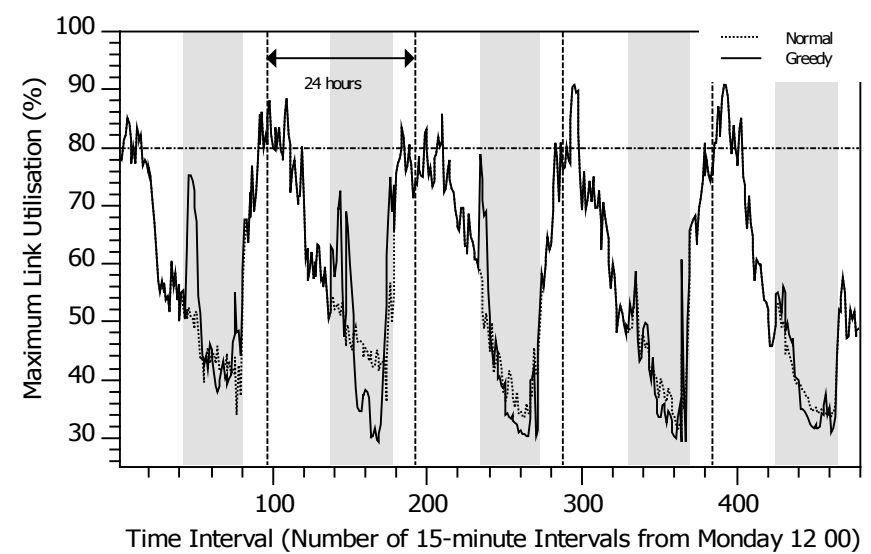

Figure 4. Variation of MLU with reduced topology of $\alpha=0.8$.

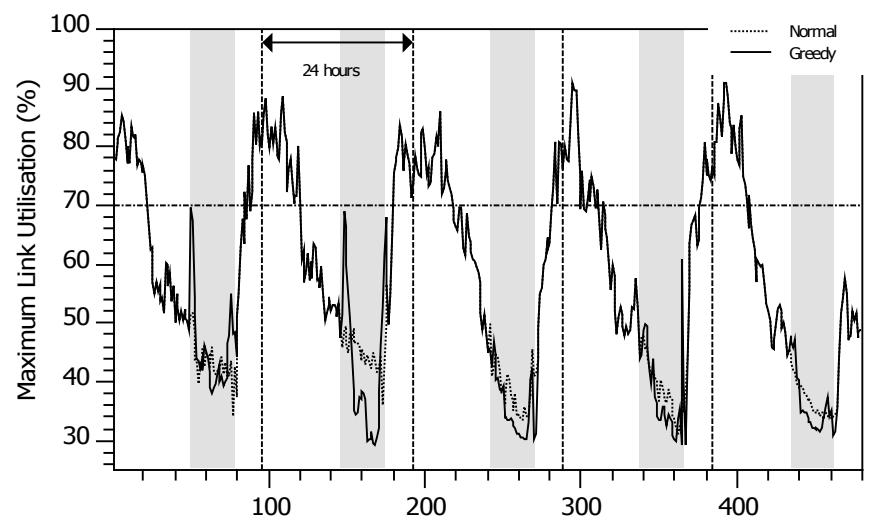

Time Interval (Number of 15-minute Intervals from Monday 1200 )

Figure 5. Variation of MLU with reduced topology of $\alpha=0.7$.

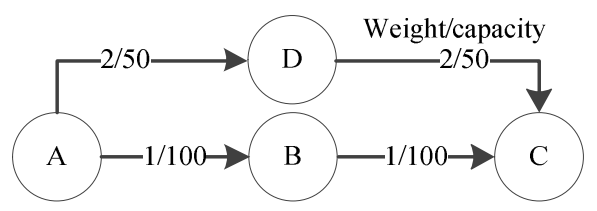

Figure 6. Illustrative network topology.

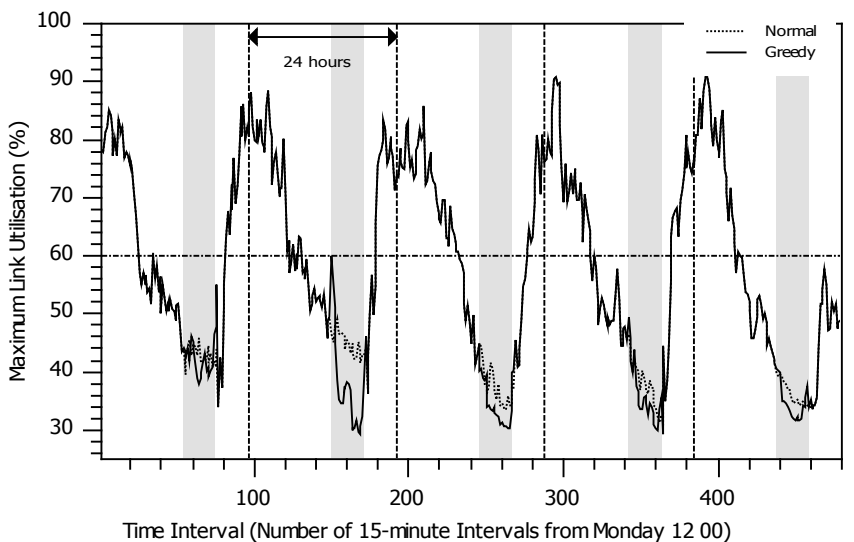

Figure 7. Variation of MLU with reduced topology of $\alpha=0.6$.

TABLE V. TRAFFIC DEMANDS AND PATHS FOR THE ILLUSTRATIVE NETWORK TOPOLOGY

\begin{tabular}{|l|l|l|l|}
\hline $\begin{array}{l}\text { Source } \rightarrow \\
\text { Destination }\end{array}$ & Demand & Initial Path & $\begin{array}{l}\text { Path after removal } \\
\text { of link A-B }\end{array}$ \\
\hline $\mathrm{A} \rightarrow \mathrm{C}$ & 10 & A-B-C & A-D-C \\
\hline $\mathrm{B} \rightarrow \mathrm{C}$ & 60 & B-C & B-C \\
\hline $\mathrm{A} \rightarrow \mathrm{D}$ & 20 & A-D & A-D \\
\hline $\mathrm{D} \rightarrow \mathrm{C}$ & 20 & D-C & D-C \\
\hline & MLU & $70 \%(B-C)$ & $60 \%(B-C)$ \\
\cline { 2 - 4 } & \multicolumn{2}{|l}{}
\end{tabular}

\section{CONCLUSION}

In this work, we presented the motivation behind developing a simple time-driven energy saving scheme for backbone networks. Our proposed heuristic algorithm determines a reduced network topology with scheduled sleeping link re-configuration and the actual off-peak time period during which this network topology is used. The scheme aims to identify an optimized trade-off between the number of sleeping links and the configuration duration for the reduced topology in order to achieve maximum energy savings performance. We demonstrated through simulations based on the GÉANT network and its actual traffic traces, that the proposed scheme is able to achieve significant energy savings in the daily operation. Furthermore, there is low impact on the end-to-end delay when applying the reduced network topology during off-peak time. Some interesting observations have also been obtained and analyzed, such as the ability of the proposed algorithm to reduce the MLU with fewer active links, which provide insights for the design of future traffic-engineering aware network optimization schemes for energy efficiency.

\section{REFERENCES}

[1] R. Bolla, R. Bruschi, K. Christensen, F. Cucchietti, F. Davoli and S. Singh, "The Potential Impact of Green Technologies in NextGeneration Wireline Networks - Is There Room for Energy Saving Optimization?" IEEE Communication Magazine (COMMAG), 2011.

[2] C. Lange, "Energy-related aspects in backbone networks," in Proceedings of 35th European Conference on Optical Communication (ECOC 2009), (Wien, AU), September 2009. 
[3] A. Greenberg, G. Hjalmtysson, D. A. Maltz, A. Myers, J. Rexford, G. Xie, H. Yan, J. Zhan, and H. Zhang, "A clean slate 4d approach to network control and management," Computer Communication Review, vol. 35, pp. 41-54, 2005.

[4] M. Baldi and Y. Ofek, "Time for a "Greener" internet," in Communications Workshops, 2009. ICC Workshops 2009. IEEE International Conference on, 2009, pp. 1-6.

[5] J. Chabarek, J. Sommers, P. Barford, C. Estan, D. Tsiang, and S. Wright, "Power awareness in network design and routing," in INFOCOM 2008. The 27th Conference on Computer Communications. IEEE, April 2008, pp. $457-465$.

[6] B. Sanso and H. Mellah, "On reliability, performance and internet power consumption," in Design of Reliable Communication Networks, IEEE DRCN'09, October 2009, pp. $259-264$.

[7] S. Nedevschi, L. Popa, G. Iannaccone, S. Ratnasamy, and D. Wetherall, "Reducing network energy consumption via sleeping and rate-adaptation," in Proceedings of the 5th USENIX Symposium on Networked Systems Design and Implementation, NSDI'08, Berkeley, CA, USA, 2008, pp. 323-336.

[8] G. Da Costa, J. P. Gelas, Y. Georgiou, L. Lefevre, A. C.Orgerie, J. M. Pierson, O. Richard, and K. Sharma, "The green-net framework: Energy efficiency in large scale distributed systems," in Parallel Distributed Processing, 2009. IPDPS 2009. IEEE International Symposium on, May 2009, pp. 1 -8.

[9] M. Gupta and S. Singh, "Greening of the internet," in SIGCOMM, 2003, pp. 19-26.

[10] A. Bianzino, C. Chaudet, D. Rossi, and J. Rougier, "A survey of green networking research," Communications Surveys Tutorials, IEEE, no. 99, pp. $1-18,2010$.

[11] R. Bolla, R. Bruschi， F. Davoli, and F. Cucchietti, "Energy Efficiency in the Future Internet: A Survey of Existing Approaches and Trends in Energy-Aware Fixed Network Infrastructures," Communications Surveys Tutorials, IEEE, no. 99, pp. $1-22,2010$.

[12] A. Cianfrani, M. Listanti, V. Eramo, M. Marazza, and E. Vittorini, "An energy saving routing algorithm for a green ospf protocol," in Proc. IEEE Infocom'10, 2010.

[13] W. Fisher, M. Suchara, and J. Rexford, "Greening backbone networks: reducing energy consumption by shutting off cables in bundled links," in Proceedings of the first ACM SIGCOMM workshop on Green networking, ser. Green Networking '10. New York, NY, USA: ACM, 2010, pp. 29-34.

[14] M. Zhang, C. Yi, B. Liu, and B. Zhang, "GreenTE: Power-Aware Traffic Engineering," in IEEE International Conference on Network Protocols (ICNP), October 2010.

[15] L. Chiaraviglio, M. Mellia, and F. Neri, "Reducing power consumption in backbone networks," in Proceedings of the 2009 IEEE international conference on Communications, ser. ICC'09. Piscataway, NJ, USA: IEEE Press, 2009, pp. 2298-2303.

[16] N. Vasic and D. Kostic, "Energy-aware traffic engineering," in Proceedings of the 1st International Conference on EnergyEfficient Computing and Networking, ser. e-Energy '10. New York, NY, USA: ACM, 2010, pp. 169-178.

[17] C. Panarello, A. Lombardo, G. Schembra, L. Chiaraviglio, and M. Mellia, "Energy saving and network performance: a trade-off approach," in Proceedings of the 1st International Conference on Energy-Efficient Computing and Networking, ser. e-Energy '10. New York, NY, USA: ACM, 2010, pp. 41-50.

[18] N. Wang, K. Ho, G. Pavlou, and M. Howarth, "An overview of routing optimization for internet traffic engineering,"
Communications Surveys Tutorials, IEEE, vol. 10, no. 1, pp. $36-$ 56 , quarter 2008.

[19] J. Restrepo, C. Gruber, and C. Machuca, "Energy Profile Aware Routing," in Communications Workshops, 2009.ICC Workshops 2009. IEEE International Conference on Green Communications, 2009, pp. $1-5$.

[20] M. Roughan, A. Greenberg, C. Kalmanek, M. Rumsewicz, J. Yates, and Y. Zhang, "Experience in measuring backbone traffic variability: models, metrics, measurements and meaning," in Proceedings of the 2nd ACM SIGCOMM Workshop on Internet measurement, ser. IMW '02. New York, NY, USA: ACM, 2002, pp. 91-92.

[21] S. Uhlig, B. Quoitin, J. Lepropre, and S. Balon, "Providing public intradomain traffic matrices to the research community," SIGCOMM Comput.Commun. Rev., vol. 36, pp. 83-86, January 2006.

[22] R. Bolla, R. Bruschi, A. Cianfrani, and M. Listanti, "Enabling backbone networks to sleep," Network, IEEE, vol. 25, no. 2, pp. 26 -31, March-April 2011.

[23] P. Psenak, S. Mirtorabi, A. Roy, L. Nguyen, and P. PillayEsnault, "Multi-Topology (MT) Routing in OSPF," RFC 4915 (Proposed Standard), Internet Engineering Task Force, June 2007.

[24] T. Przygienda, N. Shen, and N. Sheth, "M-ISIS: Multi Topology (MT) Routing in Intermediate System to Intermediate Systems (IS-ISs)," RFC 5120 (Proposed Standard), Internet Engineering Task Force, February 2008.

[25] L. Roberts, "A radical new router," Spectrum, IEEE, vol. 46, no. 7, pp. $34-39$, July 2009.

[26] M. Yamada, T. Yazaki, N. Matsuyama, and T. Hayashi, "Power efficient approach and performance control for routers," in Communications Workshops, 2009.ICC Workshops 2009. IEEE International Conference on, June 2009, pp. 1 -5.

[27] M. Baldi and Y. Ofek, "Time for a "greener" internet," in Communications Workshops, 2009. ICC Workshops 2009. IEEE International Conference on, June 2009, pp. 1 -6.

[28] R. Bolla, R. Bruschi, and A. Ranieri, "Energy-aware equipment for next-generation networks," in Proceedings of the 4th International Conference on Future Internet Technologies, ser. CFI '09. New York, NY, USA: ACM, 2009, pp. 8-11.

[29] M. Mandviwalla and N. F.Tzeng, "Energy-efficient scheme for multiprocessor-based router linecards," in Proceedings of the International Symposium on Applications on Internet. Washington, DC, USA: IEEE Computer Society, 2006, pp. 156163.

[30] L. Irish and K. Christensen, "A "Green TCP/IP" to reduce electricity consumed by computers," in Southeastcon'98. Proceedings.IEEE, April 1998, pp. $302-305$.

[31] C. Gunaratne, K. Christensen, B. Nordman, and S. Suen, "Reducing the energy consumption of ethernet with adaptive link rate (alr)," IEEE Transactions on Computers, vol. 57, pp. 448461, 2008.

[32] G. N. Rosario, G. Garroppo, Stefano Giordano, and M. Pagano, "Energy aware routing based on energy characterization of devices: Solutions and analysis," in 4th International Workshop on Green Communications (GreenComm4), Kyoto, Japan, June 2011.

[33] The TOTEM traffic engineering toolbox, [Online]. http://totem.run.montefiore.ulg.ac.be

[34] Abilene traffic matrices dataset, [Online]. Available: http://www.cs.utexas.edu/users/yzhang/research/AbileneTM 